03

\title{
Моделирование характеристик системы сверхзвуковых струй в атмосфере углекислого газа
}

\author{
(С) Ю.Н. Дерюгин ${ }^{1}$, Д.К. Зеленский ${ }^{1}$, Р.Н. Жучков ${ }^{1}$, \\ Я.В. Емельянова ${ }^{1}$, Г.А. Павлов ${ }^{2}$, А.Л. Смирнов ${ }^{2, \text { Ф }}$ \\ ${ }^{1}$ Российский федеральный ядерный центр - Всероссийский \\ научно-исследовательский институт экспериментальной фризики, Саров, \\ Россия \\ ${ }^{2}$ Институт проблем химической фризики РАН, Черноголовка, Московская \\ обл., Россия \\ ^E-mail: asm@icp.ac.ru
}

Поступило в Редакцию 2 августа 2018г.

Выполнено моделирование структуры и процессов тепломассообмена в системе выхлопных струй тормозных двигателей космических аппаратов при спуске в атмосфере Марса. Учтено, что система струй представляет собой химически реагирующую газовую среду, образованную выхлопными газами и атмосферой планеты, находится при достаточно высоких температурах и характеризуется развитой турбулентностью. Для реалистичных условий получены расчетные поля полного набора параметров среды, образованной системой взаимодействующих сверхзвуковых выхлопных струй тормозных двигателей спускаемого космического аппарата в атмосфере планеты.

DOI: 10.21883/PJTF.2018.24.47031.17489

Моделирование структуры и процессов тепломассообмена в системе выхлопных струй тормозных двигателей космических аппаратов при движении в атмосфере Марса весьма актуально в связи с планами по исследованию этой планеты, в частности с проектом „ЭкзоМарс“. Основная проблема при этом связана с необходимостью учета многих определяющих факторов, поскольку при разлете взаимодействующих сверхзвуковых струй многокомпонентная химически реагирующая газовая среда, образованная выхлопными газами и атмосферой планеты, может находиться при достаточно высоких температурах $(>1000 \mathrm{~K})$ и характеризоваться развитой турбулентностью. 
В настоящей работе выполнено трехмерное моделирование структуры четырех взаимодействующих выхлопных струй, исходящих из сопел тормозных двигателей. Параметры струй на срезе сопла и параметры окружающего газа считаются заданными (см. далее). Моделирование структуры и процессов тепломассообмена в системе струй основано на модели среды, состоящей из 19 компонентов, между которыми протекает 21 химическая реакция (константы реакций в форме Аррениуса взяты из [1]). Поскольку в поле течения реализуются невысокие давления (доли атмосферы), „ламинарные“ переносные и термодинамические характеристики многокомпонентной „идеальной“ газовой среды определены по [2,3]. Бо́льшая часть поля течения системы струй характеризуется развитой турбулентностью. При моделировании турбулентности используется несколько подходов, среди которых выделяются три основных: прямое численное моделирование (direct numerical simulation, DNS), моделирование крупных вихрей (large eddy simulation, LES) и решение осредненных по Рейнольдсу уравнений Навье-Стокса (Reynolds-averaged Navier-Stokes, RANS). В настоящее время чаще используются полуэмпирические RANS-модели турбулентности, что объясняется их быстрой сходимостью, устойчивостью к заданию краевых условий и вполне приемлемыми результатами сравнения с имеющейся экспериментальной информацией [4]. Далее для определения турбулентных характеристик (например, вязкости) используются однопараметрическая дифференциальная модель Спаларта-Алмареса (SA-модель) [5] и двухпараметрическая дифференциальная модель Ментера (SST-модель) [6].

Система уравнений, описывающая поле течения струй, взаимодействующих между собой и с атмосферой, состоит из уравнений сохранения массы, импульса и энергии, сформулированных с учетом развитой турбулентности и химической неравновесности. Система дополнена уравнением для турбулентной вязкости, SA- и SST-моделями турбулентности, граничными условиями и схемой неравновесных химических реакций. Исходная система уравнений в векторной форме имеет вид

$$
\frac{\partial \mathbf{Q}}{\partial t}+\nabla(\mathbf{F}-\mathbf{G})=\mathbf{H},
$$

где $\mathbf{Q}$ - вектор консервативных переменных, $\mathbf{F}$ - вектор конвективных потоков, $\mathbf{G}$ - вектор вязких потоков, Н - вектор источников. В качестве граничных условий заданы расположения и параметры струй на начальных участках, а также параметры среды $\mathrm{CO}_{2}$ на бесконечности.

Письма в ЖТФ, 2018, том 44, вып. 24 
Приведем схему реакций в поле течения струй, определяющих вектор источников Н:
1. $\mathrm{CO}_{2}+M_{1} \underset{K_{-1}}{\stackrel{K_{1}}{\longrightarrow}} \mathrm{CO}+\mathrm{O}+M_{1}$
2. $\mathrm{CO}+M_{2} \underset{K_{-2}}{\stackrel{K_{2}}{\longrightarrow}} \mathrm{C}+\mathrm{O}+M_{2}$,
3. $\mathrm{N}_{2}+M_{3} \underset{K_{-3}}{\stackrel{K_{3}}{\longrightarrow}} 2 \mathrm{~N}+M_{3}$,
4. $\mathrm{O}_{2}+M_{4} \underset{K_{-4}}{\stackrel{K_{4}}{\longrightarrow}} 2 \mathrm{O}+M_{4}$,
5. $\mathrm{NO}+M_{5} \underset{K_{-5}}{\stackrel{K_{5}}{\longrightarrow}} \mathrm{N}+\mathrm{O}+M_{5}$,
6. $\mathrm{CO}+\mathrm{N} \underset{K_{-6}}{\stackrel{K_{6}}{\longrightarrow}} \mathrm{NO}+\mathrm{C}$,
7. $\mathrm{CO}+\mathrm{CO} \underset{K_{-7}}{\stackrel{K_{7}}{\longrightarrow}} \mathrm{CO}_{2}+\mathrm{C}$,
8. $\mathrm{CO}+\mathrm{O} \underset{K_{-8}}{\stackrel{K_{8}}{\longrightarrow}} \mathrm{O}_{2}+\mathrm{C}$,
9. $\mathrm{N}_{2}+\mathrm{O} \underset{K_{-9}}{\stackrel{K_{9}}{\longrightarrow}} \mathrm{NO}+\mathrm{O}$,
10. $\mathrm{NO}+\mathrm{CO} \underset{K_{-10}}{\stackrel{K_{10}}{\longrightarrow}} \mathrm{CO}_{2}+\mathrm{N}$,
11. $\mathrm{NO}+\mathrm{O} \underset{K_{-11}}{\stackrel{K_{11}}{\longrightarrow}} \mathrm{O}_{2}+\mathrm{N}$,
12. $\mathrm{CO}_{2}+\mathrm{O} \underset{K_{-12}}{\stackrel{K_{12}}{\longrightarrow}} \mathrm{O}_{2}+\mathrm{CO}$,
13. $\mathrm{H}_{2}+M \underset{K_{-13}}{\stackrel{K_{13}}{\longrightarrow}} 2 \mathrm{H}+M$,
14. $\mathrm{H}+\mathrm{OH}+M \underset{K_{-14}}{\stackrel{K_{14}}{\longrightarrow}} \mathrm{H}_{2} \mathrm{O}+M$,
15. $\mathrm{O}_{2}+\mathrm{H} \underset{K_{-15}}{\stackrel{K_{15}}{\longrightarrow}} \mathrm{OH}+\mathrm{O}$,
16. $\mathrm{CO}+\mathrm{OH} \underset{K_{-16}}{\stackrel{K_{16}}{\leftrightarrows}} \mathrm{H}+\mathrm{CO}_{2}$,
17. $\mathrm{N}+\mathrm{O} \underset{K_{-17}}{\stackrel{K_{17}}{\longrightarrow}} \mathrm{NO}^{+}+e$,
18. $\mathrm{O}+\mathrm{O} \underset{K_{-18}}{\stackrel{K_{18}}{\longrightarrow}} \mathrm{O}_{2}^{+}+e$,
19. $\mathrm{C}+\mathrm{O} \underset{K_{-19}}{\stackrel{K_{19}}{\longrightarrow}} \mathrm{CO}^{+}+e$,
20. $\mathrm{O}+e \underset{K_{-20}}{\stackrel{K_{20}}{\longrightarrow}} \mathrm{O}^{+}+2 e$,
21. $\mathrm{C}+e \underset{K_{-21}}{\stackrel{K_{21}}{\longrightarrow}} \mathrm{C}^{+}+2 e$.

Вычислительные алгоритмы расчета параметров поля течения основаны на методе расщепления по физическим процессам, конечнообъемном методе на неструктурированных сетках и неявном методе интегрирования. Счетный шаг расщепляется на три этапа. На первом этапе решаются уравнения Навье-Стокса в предположении замороженных турбулентности и кинетики химических реакций. Уравнения Навье-Стокса аппроксимированы с помощью метода конечного объема на неструктурированной сетке. Полученная система нелинейных разностных уравнений решается итерационным методом Ньютона относительно приращения решения. В разностной схеме явная аппроксимация конвективных потоков основана на подходе Годунова [7] второго порядка точности и приближенном методе Роу [8] для решения задачи Римана о распаде разрыва. Чтобы повысить порядок аппроксимации до второго, проводится реконструкция решения, заключающаяся в определении параметров слева и справа от грани расчетной ячейки. Реконструкция проводится для примитивных параметров $\varphi=\{\rho, u, v, w, p\}$. Значение градиента поля $(\operatorname{grad} \varphi)_{p}$, используемое при реконструкции, опреде-

Письма в ЖТФ, 2018, том 44, вып. 24 


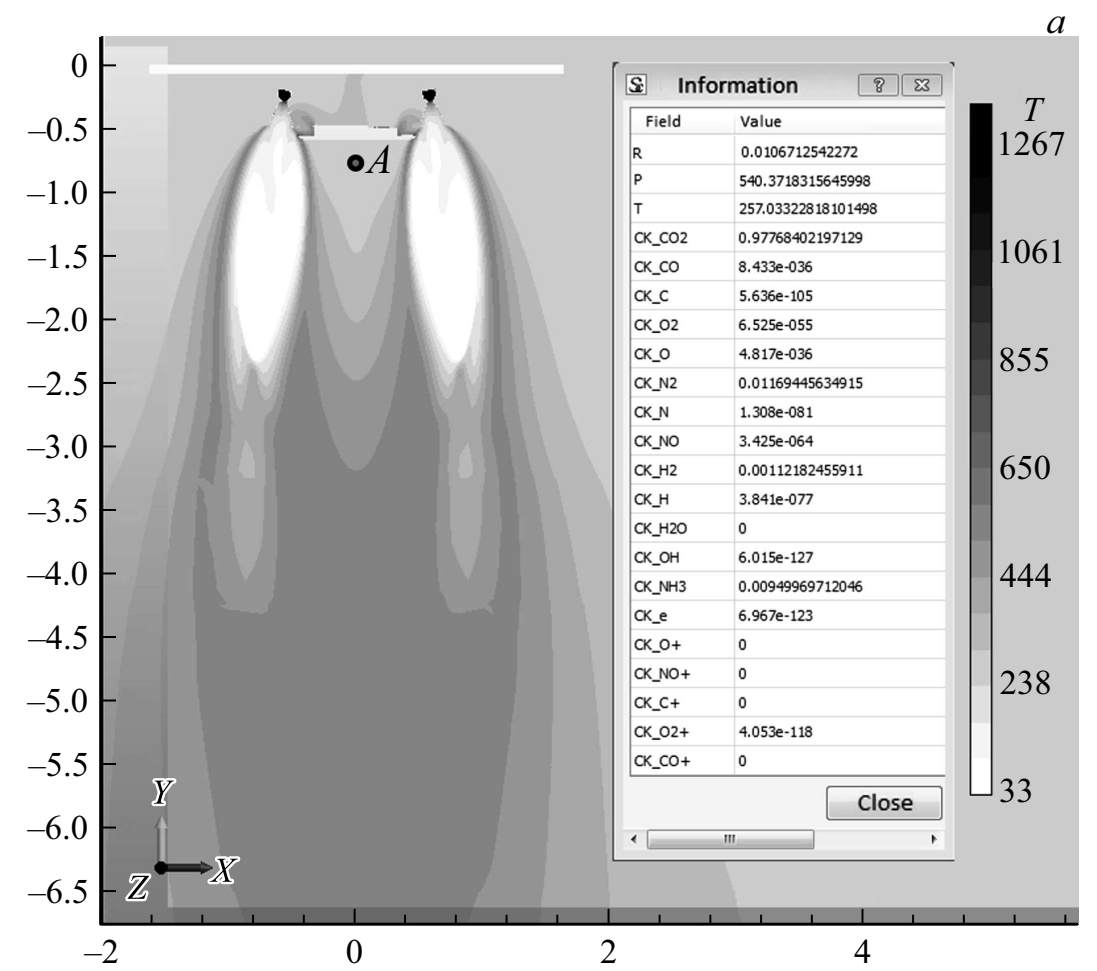

Поле значений температуры на срезе в системе выхлопных струй тормозных двигателей. Встроены таблицы значений параметров поля течения в расчетных точках $A, B$ и $C: a-$ в придонной области космического аппарата (точка $A$ ), $b-$ в начале зоны смешения выхлопных струй (точка $B$ ), $c-$ в середине зоны смешения выхлопных струй (точка $C$ ). Значения параметров (плотность $R$, давление $P$, температура $T$ ) даны в системе СИ, концентрации компонентов в массовых долях, координаты по осям - в метрах.

ляется методом наименьших квадратов [9] с использованием ограничителя потока в соответствии с [10]. Неявная аппроксимация невязкого потока в левой части уравнения для приращения консервативных переменных $Q$ связана с расщеплением потоков в зависимости от собственных чисел матрицы Якоби. Конвективные потоки на внешних гранях определяются в зависимости от поставленных граничных усло-

Письма в ЖТФ, 2018, том 44, вып. 24 


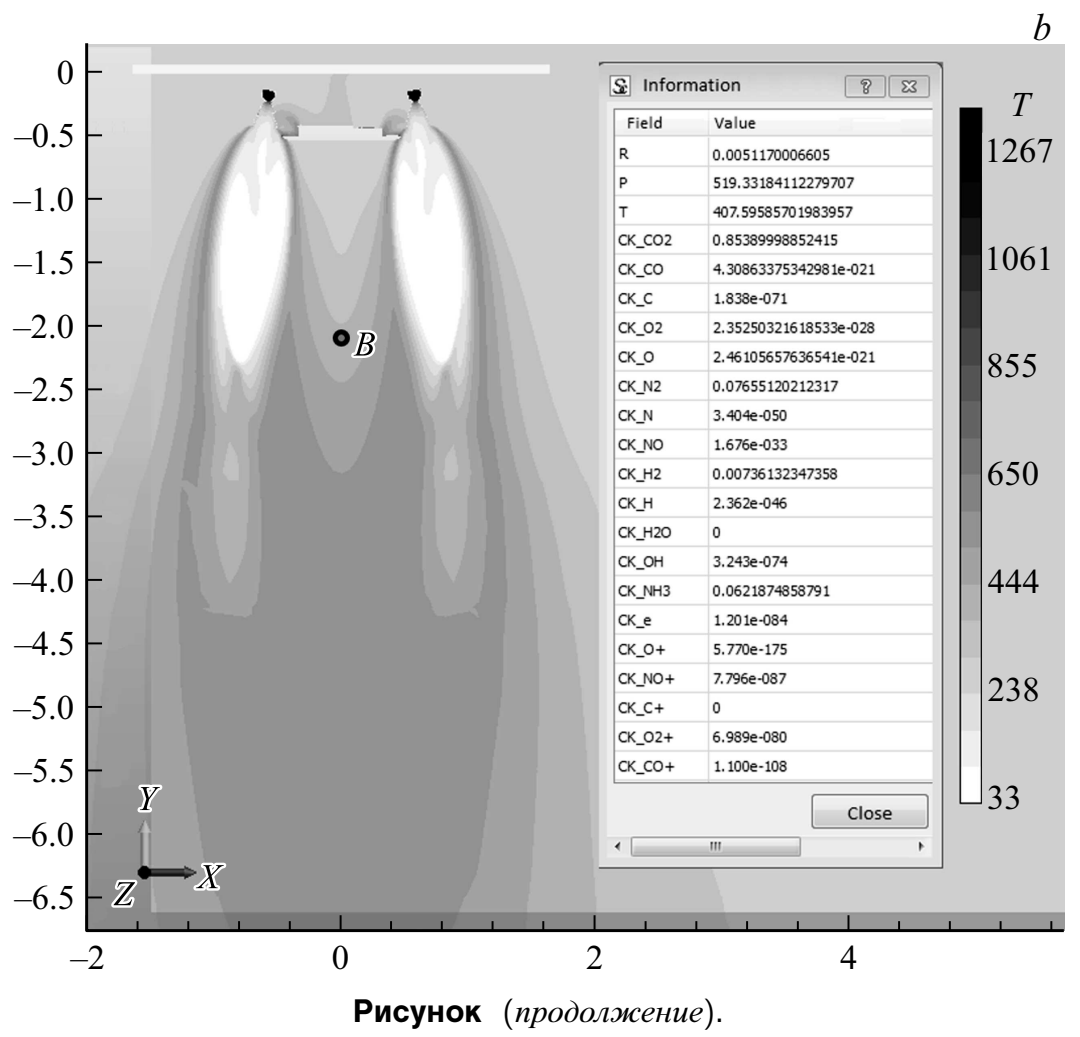

вий. Недостающие граничные условия на внешних границах расчетной области дополняются соотношениями для характеристик, приходящих на границу из расчетной области [11]. Для явного и неявного определения диффузионных потоков используется центрально-разностная аппроксимация. Смешанные производные в центре грани определяются интерполяцией от компонент градиентов, определенных в центрах ячеек. При решении уравнений Навье-Стокса для течений среды, состоящей из $N$ компонентов, коэффициентами разностных уравнений являются квадратные матрицы размерности $((5+N) \times(5+N))$. Для решения системы линейных алгебраических уравнений используется алгебраический многосеточный параллельный решатель AMG [12].

Письма в ЖТФ, 2018, том 44, вып. 24 


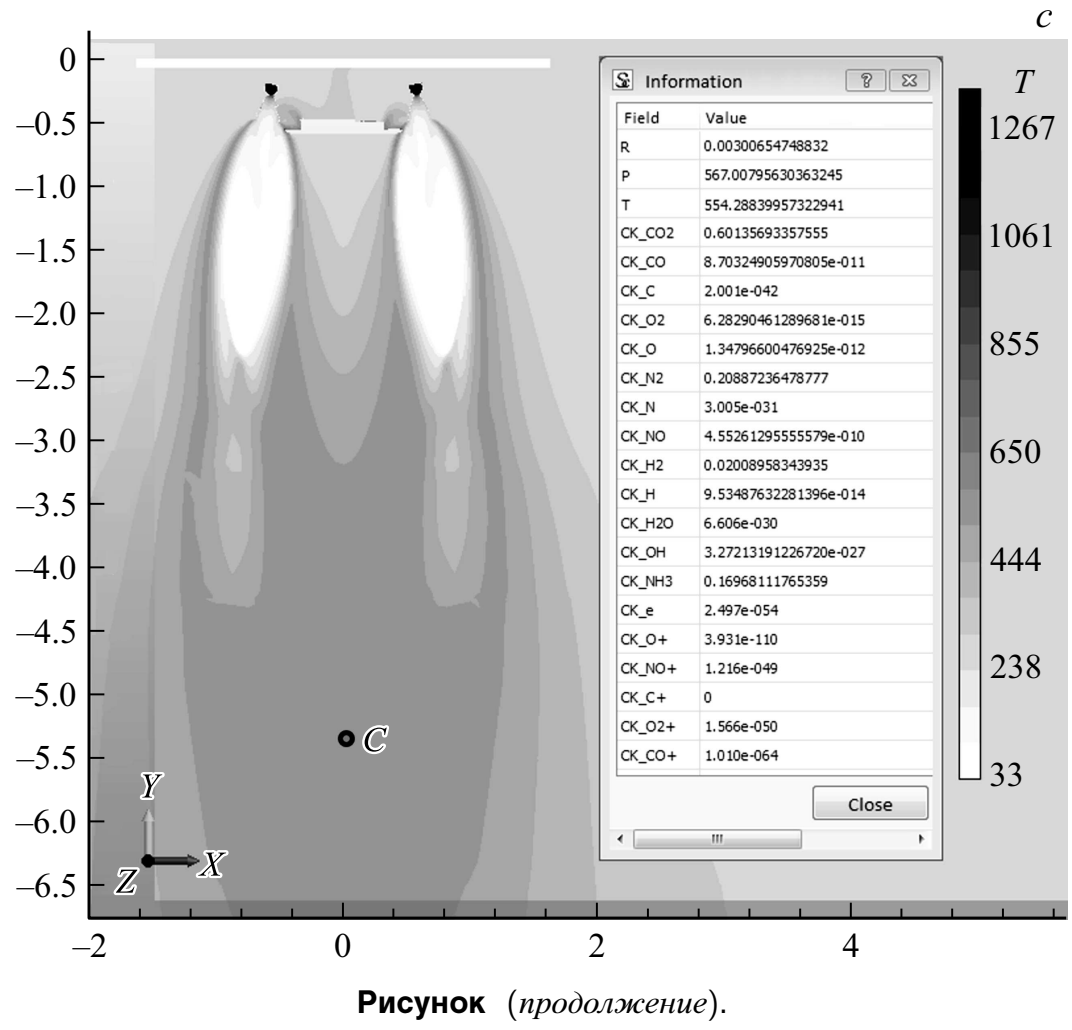

На втором этапе решаются уравнения химической кинетики $[13,14]$. Численный метод решения уравнений химической кинетики основан на разностной схеме Розенброка [15]. При расчете химических реакций в турбулентном потоке производится оценка времени турбулентного смешения по турбулентным параметрам потока с помощью STT-модели турбулентности. В качестве скорости реакции выбирается минимальная скорость между скоростью турбулентного смешения и скоростью самой реакции.

На заключительном (третьем) этапе определяется изменение турбулентных параметров потока за счет генерации и диссипации турбулентности. В общем случае каждое из уравнений модели турбулентности 
сводится к решению обыкновенных дифференциальных уравнений. Входящие в определение генерационного и диссипативного членов уравнения значения градиента от параметров потока вычисляются в центре ячейки по формуле Грина-Гаусса. Уравнение аппроксимируется явно-неявной разностной схемой, которая обеспечивает положительность решения. Данная методика реализована в рамках пакета программ ЛОГОС [16].

В работе выполнено трехмерное моделирование структуры четырех взаимодействующих выхлопных струй, исходящих из сопел тормозных двигателей. Для модельного расчета выбраны следующие довольно реалистичные условия: диаметр сопел на срезе $0.2 \mathrm{~m}$, сопла равномерно расположены на окружности диаметром $1.2 \mathrm{~m}$, струи на срезе сопел состоят из смеси газов при давлении $4450 \mathrm{~Pa}$, температуре $337 \mathrm{~K}$, массовой скорости $2275 \mathrm{~m} / \mathrm{s}$, числе Маха 4.5, мольной доле $\mathrm{H}_{2}-0.364$, $\mathrm{N}_{2}-0.272, \mathrm{NH}_{3}-0.364$. Установившееся течение около летательного аппарата определялось в результате решения нестационарных уравнений с неизменными граничными параметрами набегающего потока при стремлении $t \rightarrow \infty$. Расчеты проводились в обращенной постановке, когда обтекаемое тело считается неподвижным. Скорость набегающего потока соответствует скорости полета аппарата, которая принималась равной $45 \mathrm{~m} / \mathrm{s}$. Параметры атмосферы (набегающего потока) следующие: давление $1000 \mathrm{~Pa}$, температура $288 \mathrm{~K}$. Выполнены расчеты с учетом химических реакций [1]. Результаты расчетов показаны на рисунке, где приведены расчетные поля параметров системы взаимодействующих сверхзвуковых выхлопных струй тормозных двигателей спускаемого космического аппарата в атмосфере Марса. Информация о полях параметров, в частности, является ключевой для анализа характеристик взаимодействия СВЧ-излучения с системой факелов выхлопных струй тормозных двигателей спускаемого аппарата.

\section{Список литературы}

[1] Troschiev Yu.V., Pavlov G.A. // Int. J. Heat Mass Transfer. 2016. V. 99. P. 234-242; Equist K.T., Hilles B.R., Jonston C.D., Bose D., White T.R., Mahzari M. // J. Spacecraft Rockets. 2014. V. 54. N 4. P. 1106-1124; Суржиков С.Т. // Физ.-хим. кинетика в газовой динамике. 2015. Т. 16. В. 2. C. $1-74$.

[2] Гиршфельдер Джс., Кертисс Ч., Берд Р. Молекулярная теория газов и жидкостей. М.: ИЛ, 1961. 929 с.

5 Письма в ЖТФ, 2018, том 44, вып. 24 
[3] Алексеев Б.В. Математическая кинетика реагирующих газов. М.: Наука, 1982. $422 \mathrm{c}$

[4] Волков К.Н., Емельянов В.Н. Моделирование крупных вихрей в расчетах турбулентных течений. М.: Физматлит, 2008. 364 с.

[5] Spalart P.R., Allmaras S.R. A one-equation turbulence model for aerodynamic flows // Proc. 30th Aerospace Sciences Meeting and Exhibition. Reno, Nevada, USA, 1992. Paper N AIAA 92-0439.

[6] Menter F.R. Zonal two equation $\mathrm{k}-\omega$ turbulence models for aerodynamic flows // Proc. 24th Fluid Dynamics Conf. Orlando, Florida, USA, 1993. Paper N AIAA 93-2906.

[7] Годунов С.К. // Мат. сб. 1959. Т. 47(89). № 3. С. 271-306.

[8] Roe P.L. // J. Comput. Phys. 1983. V. 49. N 6. P. 357-393.

[9] Мелешкин Н.В., Дерюгин Ю.Н., Зеленский Д.К. Пакет программ ЛОГОС. Численное исследование точности аппроксимации дифференциальных операторов на различных сетках // Tp. XIV Междунар. конф. „Супервычисления и математическое моделирование“. Саров, 2013. С. 408-415.

[10] Venkatakrishnan $V$. On the accuracy of limiters and convergence to steady state solutions // Technical Report. American Institute of Aeronautics and Astronautics, 1993. Paper N AIAA-93-0880.

[11] Giles M.B. // AIAA Journal. 1990. V. 28. N 12. P. 2050-2058.

[12] Волков К.Н., Дерюгин Ю.Н., Емельянов В.Н., Карпенко А.Г., Козелков А.С., Тетерина И.В. Методы ускорения газодинамических расчетов на неструктурированных сетках. М.: Физматлит, 2013. 536 с.

[13] Spalding D.B. Mixing and chemical reaction in steady confined turbulent flame // Proc. 13th Int. Symp. on Combustion. Pittsburgh, USA, 1971. P. 649657; Иевлев В.М. Турбулентное движение высокотемпературных сплошных сред. М.: Наука, 1975. 256 с.

[14] Magnussen B.F. On the structure of turbulence and a generalized eddy dissipation concept for chemical reaction in turbulent flow // 19th American Institute of Aeronautics and Astronautics Aerospace Science Meeting. St. Louis, USA, 1981. DOI: 10.2514/6.1981-42

[15] Хайрер Э., Ваннер Г. Решение обыкновенных дифференциальных уравнений. Жесткие и дифференциально-алгебраические задачи. М.: Мир, 1999. $685 \mathrm{c}$.

[16] Козелков А.С., Дерюгин Ю.Н., Зеленский Д.К., Глазунов В.А., Голубев А.А., Денисова О.В., Лашкин С.В., Жучков Р.Н., Тарасова Н.В., Сизова М.А. Многофункциональный пакет программ ЛОГОС для расчета задач гидродинамики и тепломассопереноса на многопроцессорных ЭВМ: базовые технологии и алгоритмы // Тр. ХІІ Междунар. семинара „Супервычисления и математическое моделирование“. Саров, 2010. С. 215-230.

Письма в ЖТФ, 2018, том 44, вып. 24 